\title{
Acoustics Research of Propulsion Systems
}

\author{
Ximing Gao and Janice D. Houston. ${ }^{2}$ \\ NASA Marshall Space Flight Center, Huntsville, AL, 35812
}

\begin{abstract}
The liftoff phase induces some of the highest acoustic loading over a broad frequency for a launch vehicle. These external acoustic environments are used in the prediction of the internal vibration responses of the vehicle and components. Thus, predicting these liftoff acoustic environments is critical to the design requirements of any launch vehicle but there are challenges. Present liftoff vehicle acoustic environment prediction methods utilize stationary data from previously conducted hold-down tests; i.e. static firings conducted in the 1960's, to generate 1/3 octave band Sound Pressure Level (SPL) spectra. These data sets are used to predict the liftoff acoustic environments for launch vehicles. To facilitate the accuracy and quality of acoustic loading, predictions at liftoff for future launch vehicles such as the Space Launch System (SLS), non-stationary flight data from the Ares I-X were processed in PC-Signal in two forms which included a simulated hold-down phase and the entire launch phase. In conjunction, the Prediction of Acoustic Vehicle Environments (PAVE) program was developed in MATLAB to allow for efficient predictions of sound pressure levels (SPLs) as a function of station number along the vehicle using semiempirical methods. This consisted, initially, of generating the Dimensionless Spectrum Function (DSF) and Dimensionless Source Location (DSL) curves from the Ares I-X flight data. These are then used in the MATLAB program to generate the $1 / 3$ octave band SPL spectra. Concluding results show major differences in SPLs between the hold-down test data and the processed Ares I$\mathrm{X}$ flight data making the Ares I-X flight data more practical for future vehicle acoustic environment predictions.
\end{abstract}

\section{Nomenclature}

$\begin{array}{ll}c & =\text { Speed of sound } \\ d_{e_{e f f}} & =\text { Effective Nozzle Diameter, } \mathrm{ft} \\ D S F & =\text { Dimensionless Spectrum Function } \\ D S L & =\text { Dimensionless Source Location } \\ F c & =\text { Center band frequency, } \mathrm{Hz} \\ I O P & =\text { Ignition Overpressure } \\ L O A & =\text { Liftoff Acoustic } \\ R & =\text { Distance from noise source to observation point } \\ S P L & =\text { Sound Pressure Level, } \mathrm{dB} \\ S t & =\text { Strouhal Number } \\ T T & =\text { Total Thrust of Engine, } \mathrm{lbf} \\ p & =\text { Pressure, psi } \\ \rho & =\text { Density, lb/f스 } 3 \\ V_{e} & =\text { Exhaust Velocity, } \mathrm{ft} / \mathrm{s} \\ X & =\text { Station Number, } \mathrm{ft} \\ X_{0} & =\text { Distance from source to nozzle, } \mathrm{ft}\end{array}$

\section{Introduction}

A coustic loads are spatially and frequency dependent sound pressure fluctuations on vehicle surfaces as a consequence of turbulent mixing of the engine exhaust. Space launch vehicles are subjected to significant amounts of fluctuating pressure loads externally during its propulsion system's period of operation. In particular the Liftoff Acoustics (LOA) of a vehicle's flight phase induces some of the highest frequencies and is considered a

\footnotetext{
${ }^{1}$ Intern, ER42, Marshall Space Flight Center, Rutgers University

${ }^{2}$ Flight Vehicle Acoustics Engineer, ER42, Marshall Space Flight Center
} 
critical design factor in the development of a launch vehicle. Since excessive magnitudes of LOA loading on a vehicle can result in malfunctions of mechanical or electronic components as well as structural fatigue, a thorough analysis of the acoustic loading at this particular phase is imperative to the prediction of vibration loads experienced by the vehicle as well as providing a basis for the development of vibration test specifications.

A continuous effort is dedicated towards the improvement of LOA loading predictions that would eventually set certain design criteria for vibroacoustic analysts to generate corresponding qualification environments for vehicle components. To facilitate these efforts, a semi-empirical prediction program known as Prediction of Acoustic Vehicle Environments (PAVE) was developed in MATLAB Graphical User Interface (GUI) format to allow for quick and user friendly predictions of sound pressure levels (SPL) experienced at launch based on existing data.

Current prediction methods utilizing stationary data from previously conducted hold-down tests tend to under predict SPL values at later time frames within the vehicle's launch phase due to effects of impingement. Since the information referenced for semi-empirical predictions from hold-down tests are considered stationary data while the liftoff phase of a rocket is inherently non-stationary, current prediction methods require a correction factor (approximately $5 \mathrm{~dB}$ ) to be added to stationary based predictions to account for impingement effects. To provide more accurate and efficient SPL predictions for current and future launch vehicle programs, non-stationary flight data from the Ares I-X launch of 2009 was processed to be used in conjunction with PAVE. As LOA conditions tend to vary throughout the launch phase, two sets of data were processed to provide predictions for a simulated hold-down phase, as well as an entire launch phase.

\section{General Methodology}

LOA environments are regularly analyzed and predicted in the form of Sound Pressure Levels (SPL) in units of decibels (dB). The SPL is described as a function of center band frequency, independent of time, and is proportional to a number of conditions that are dependent on the type of rocket analyzed. The SPL of a rocket can be computed through Eq. 1 below:

$$
S P L(F C)=10 \log _{10}(D S F)+10 \log _{10}\left[\frac{2.685 * T T * d_{e} e f f}{2}\right]+10 \log _{10}(0.231 * F C)-20 \log _{10}(R)+127.5
$$

where DSF is the dimensionless spectrum function, TT is the total thrust, $d_{e_{e f f}}$ is the effective exit diameter, and $\mathrm{Fc}$ is the center band frequency. $\mathrm{R}$ represents the coordinate system of the rocket and is equivalent to the sum of the station number $\mathrm{X}$ and the distance from the nozzle to the noise source $\mathrm{X}_{0}$ as shown in Fig.1. In particular, $\mathrm{X}_{0}$ is proportional to another critical factor in determining the SPL known as the Dimensionless Source Location or DSL. $\mathrm{X}_{0}$ is equivalent to the product between the DSL and the effective diameter of the rocket engine.

\section{A. Dimensionless Spectrum Function (DSF)}

A particular parameter required in the determination of SPL is the dimensionless spectrum function or DSF. This dimensionless parameter is defined by the equation below:

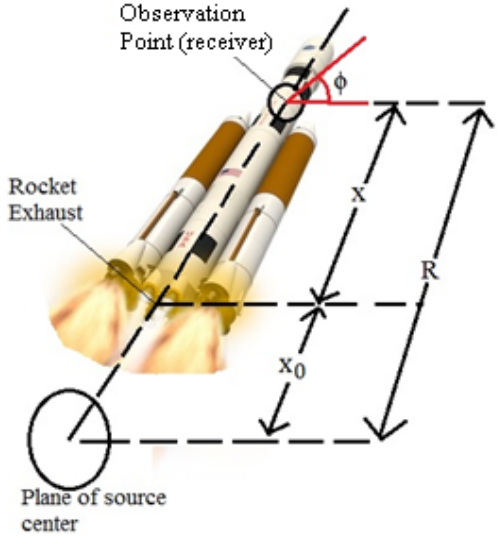

Figure 1. Rocket Coordinate System

$$
D S F=A(f)\left[\frac{v}{d_{e_{e f f}} \rho c W}\right]
$$

where $A(f)$ is the spectrum function, $v$ is velocity, $\rho$ is the density, $c$ is the speed of sound, and $W$ is mechanical power. Specifically, the spectrum function, A(f), results when a small portion of the total mechanical power of a jet is converted into turbulent power which itself, has a certain amount converted to sound power. Thus, $A(f)$ appears as a form of "spectrum density" proportional to sound power and can be determined directly as pressure squared per unit frequency as shown below.

$$
A(f)=\frac{p^{2}}{f}
$$

where $\mathrm{p}$ is pressure and $\mathrm{f}$ is frequency measured in Hertz. The spectrum function is essentially the data in normalized, spectrum level form with the bandwidth removed. This is due to the fact that the spectrum function is defined in units per Hertz and is thus in narrow band form. The data can then be non-dimensionalized using Eq. 2, 
forming the dimensionless spectrum function. It is however, commonly preferred that the SPL be viewed in $1 / 3$ octave bandwidth, thus it is shown in Eq. 1 that the 1/3 octave bandwidth must inserted back into the calculation through the multiple of .231; the center band frequency.

\section{B. Dimensionless Source Location (DSL)}

The SPL magnitude as was shown in Fig. 1 is also location dependent. Specifically, the magnitude of SPL is expected to decrease as the distance between the observation point and noise source increases. A range of dimensionless values known as dimensionless source locations or DSL, are required in the determination of the sum $\mathrm{R}$, of the station number and the distance from the nozzle to the noise source. The DSL defines the distance from the source to the receiver and is inversely proportional to the Strouhal number. It can be determined through Eq. 4 below.

$$
D S L=\frac{X_{0}}{d_{e_{e f f}}}
$$

where $\mathrm{X}_{0}$ is the distance from the nozzle to the noise source and $d_{e_{e f f}}$ is the effective exit diameter.

\section{Prediction of Acoustic Vehicle Environments (PAVE) Program}

To facilitate the understanding of LOA environments and effectively predict sound pressure levels for a wide variety of current and future launch vehicles, the Prediction of Acoustic vehicle Environments (PAVE) program was developed in a user-friendly MATLAB Graphical User Interface (GUI) format. PAVE is a 1-D semiempirical based prediction program developed using similar methods to a predecessor program written in BASIC format known as the Vehicle Acoustic Environment Prediction Program (VAEPP). PAVE is designed to

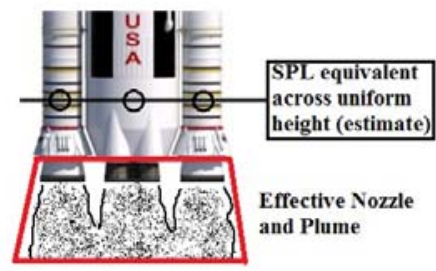

Figure 3. Engine Clusters determine the SPL at locations on the launch vehicle itself, and is thus a 1-D program that assumes acoustic sources in a single vertical axis below the rocket even though the plume of the rocket is affected by a deflector. These assumptions are displayed in Fig. 2. Furthermore, under conditions where multiple engines are in a cluster, the effective nozzle diameter may be calculated by taking the product between the exit diameter and the square root of the number of engines. This assumption is reasonable when the microphone is placed at a location downstream of the rocket plume where the individual plumes from the nozzles have mixed and can be considered as a single source (see Fig. 3).

Similar to its predecessor, PAVE utilizes a combination of user defined inputs (engine diameter, exit velocity, etc.) and empirical data to obtain values for the sound pressure levels experienced by the launch vehicle as shown in Fig. 4. PAVE's features allows for the user to choose any (realistic) frequency range (in $1 / 3$ octave bandwidth) for their calculations

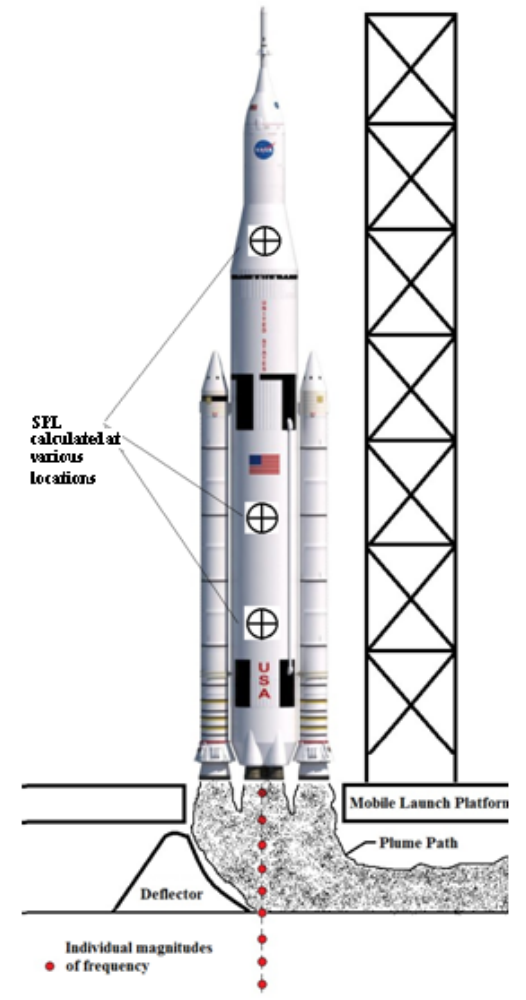

Figure 2. Apparent Sources and SPL by inputting a lower and upper frequency value. The program is configured to define a set of band numbers that covers an exact center frequency range from 1.26 to $100000 \mathrm{~Hz}$. In addition, PAVE is capable of simultaneously performing SPL predictions at five separate station locations on the rocket and displaying the results on a graph and spreadsheet. These features can be visualized in Fig. 5 below.

PAVE's prediction methods operate by extracting and interpolating/extrapolating from existing stationary and non-stationary engine data based on the engine type the user selects. Thus, all vehicle data is stored and extracted from a central excel file. Because the data of the acoustic environment is in log scale, the interpolation process must also be completed in log scale. Specifically, the data in $\log -\log$ form is linear to a degree to which linear interpolation can be executed relatively accurately. To facilitate this process, the PAVE program reads in the data in an already converted log form from excel for Strouhal number, DSL, and DSF, then conducts linear interpolation and extrapolation as necessary. The anti-log is then taken of the new interpolated/extrapolated values to calculate the 
SPL. In order to prevent the generation of imaginary values at high Strouhal numbers, new values based on extremely high Strouhal numbers as high as 100 (that will theoretically not be considered in testing) were entered into the excel spread sheet along with their corresponding DSL and DSF values, forcing the program to interpolate linearly based on a specified point.

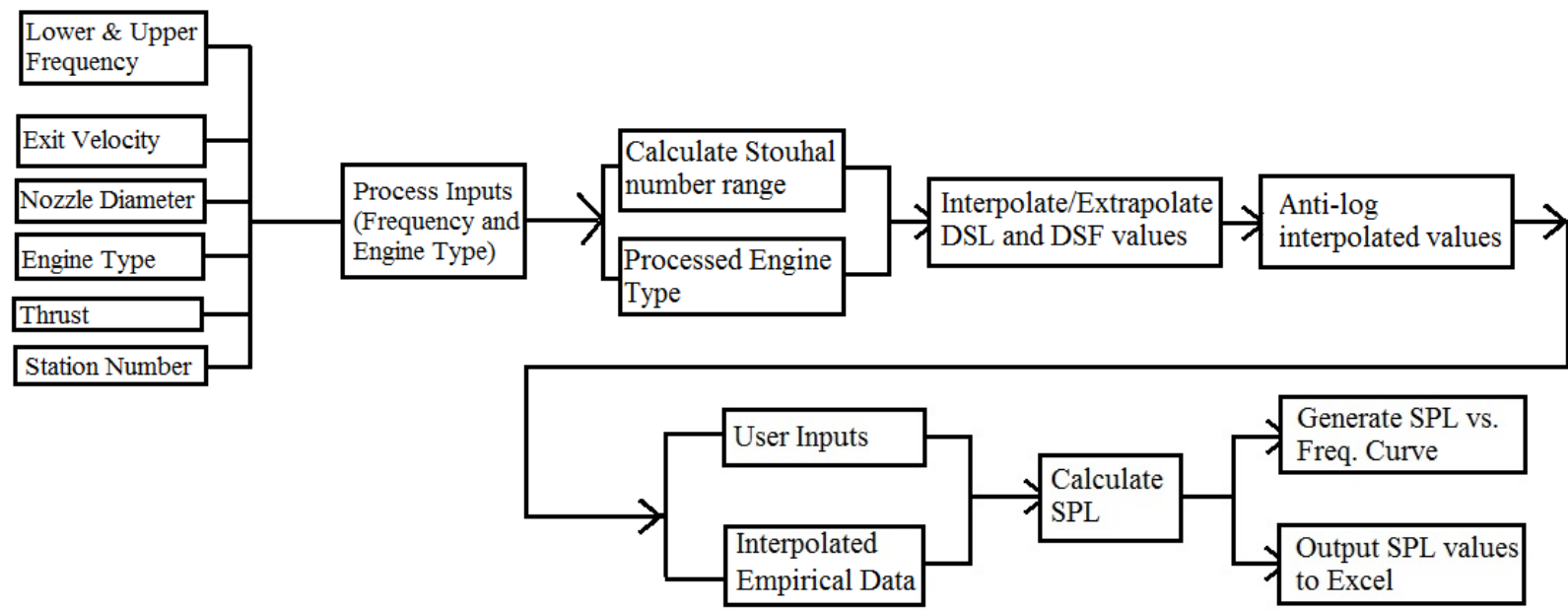

Figure 4. PAVE Program Block Diagram

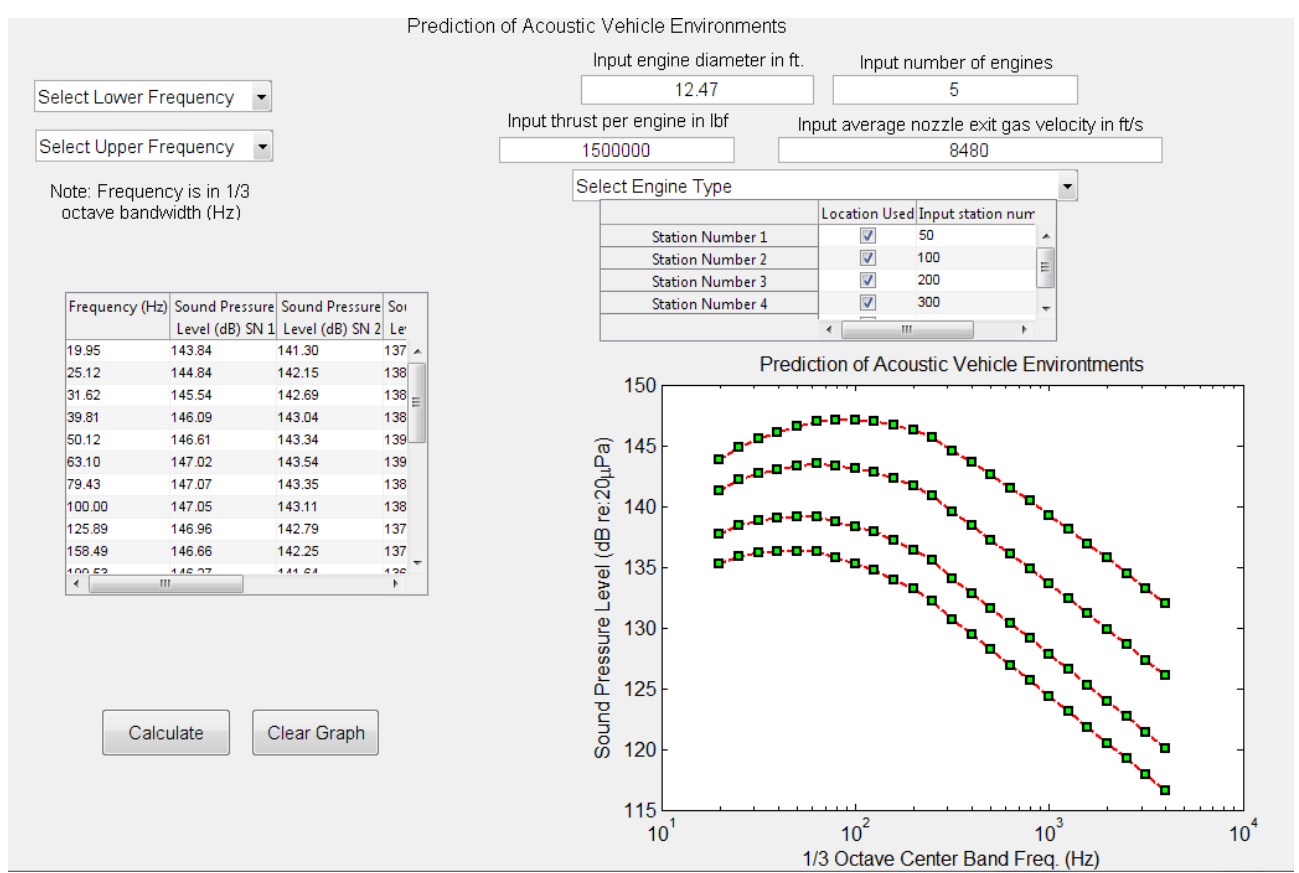

Figure 5. PAVE Graphical User Interface

\section{Ares I-X Processed Flight Data}

Ares I-X was instrumented with pressure transducers to measure the acoustic environments during its launch on October 28, 2009. The availability of flight data as well as effective methods for acoustic prediction served as incentives for the development of dimensionless spectral function (DSF) and dimensionless source location (DSL) curves for the Ares I-X rocket. Specifically the use of flight data would allow for a wide range of relatively reliable predictions using semi-empirical methods (in conjunction with PAVE), including the effects of impingement, which would have otherwise been unrealistic with hold-down test data. The project objective was to develop two pairs of DSF and DSL curves that represented both a simulated hold-down phase (period between the end of IOP and impingement effects) as well as the entire launch phase. 


\section{A. Simulated Hold-down Phase}

The Ares I-X Flight Data includes highly non-stationary time signals. Consequently, the ideal method for processing the $1 / 3$ octave band spectra analyzes each sensor via instantaneous time signals per band. Each sensor is analyzed in a range of approximately 3 seconds with a set of 4 octave spectras and covering frequency ranges of $19.95 \mathrm{~Hz}$ to $3981.07 \mathrm{~Hz}$. The time average was selected to be 1 second for all cases and the starting time was selected at 0.66 seconds, clearing the IOP range. The time ranges covered for each sensor were from 0.66 to $1.66,1$ to $2,1.5$ to 2.5 , and 2.5 to 3.5 seconds, and were reasonably selected based on the times of IOP and impingement occurrence analyzed in the wideband time history (identified to maintain realistic hold-down conditions).

The first set of data processed was for sensor AAD158P. This sensor was placed at the aft skirt of the Ares-IX and naturally experiences some of the highest frequencies due to its close proximity with the exhaust. The results are shown in Fig. 6 below.

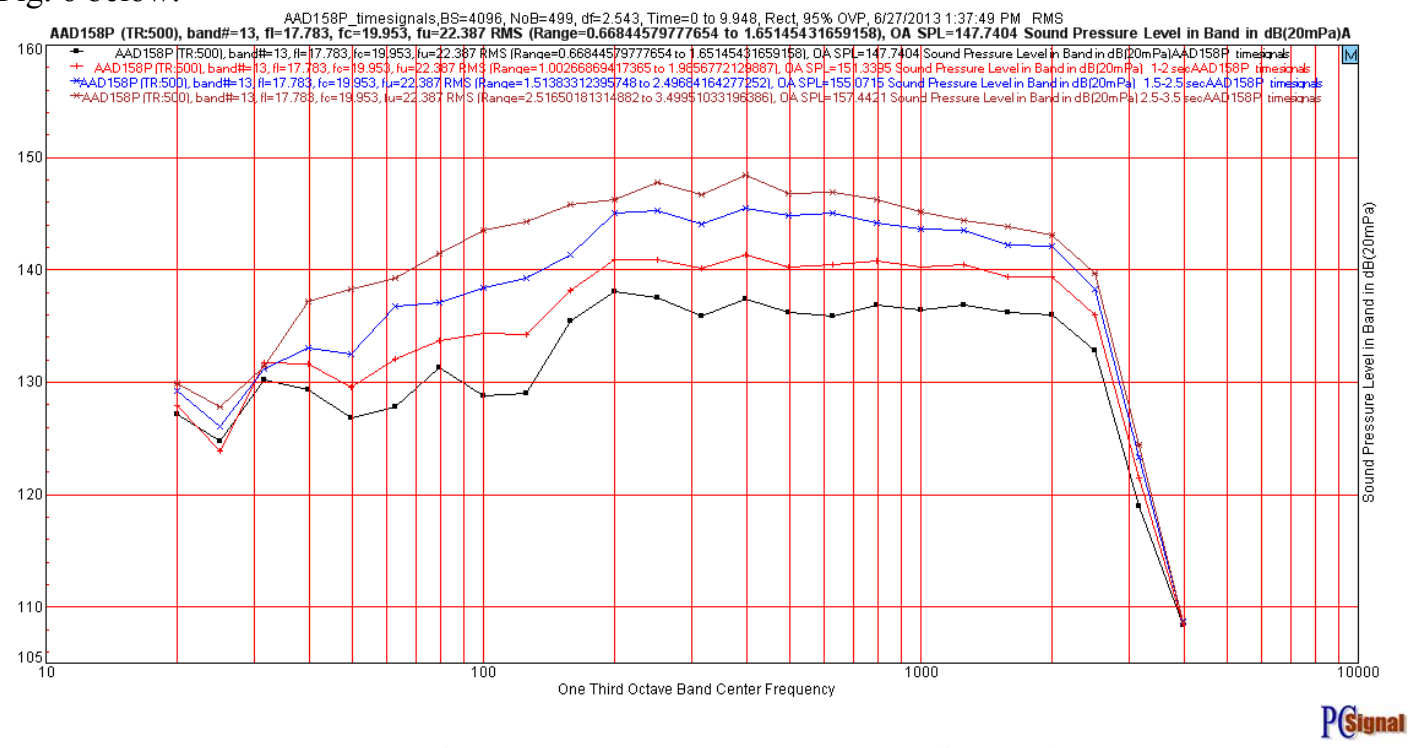

Figure 6. Octave Spectras Processed for AAD158P Sensor (aft skirt)

The octave spectrum shows a dramatic increase in overall SPL on the order of $10 \mathrm{~dB}$ from $147.74 \mathrm{~dB}$ at the 0.66 1.66 seconds time average to $157.44 \mathrm{~dB}$ at the 2.5-3.5 seconds time average. The high SPL value at the 2.5-3.5 second range suggests that the liftoff phase is approaching the effects of impingement, and is thus, not ideal for hold-down analysis. Furthermore, the short time span from hold-down to impingement suggests a quick overall liftoff phase for the Ares-IX. Ultimately, the 1.5 to 2.5 second range was found to be an ideal time range in all sensors where the effects of IOP have ended and impingement influences have yet to occur. This can also be observed in the time signals for each channel as shown in Fig. 7 below

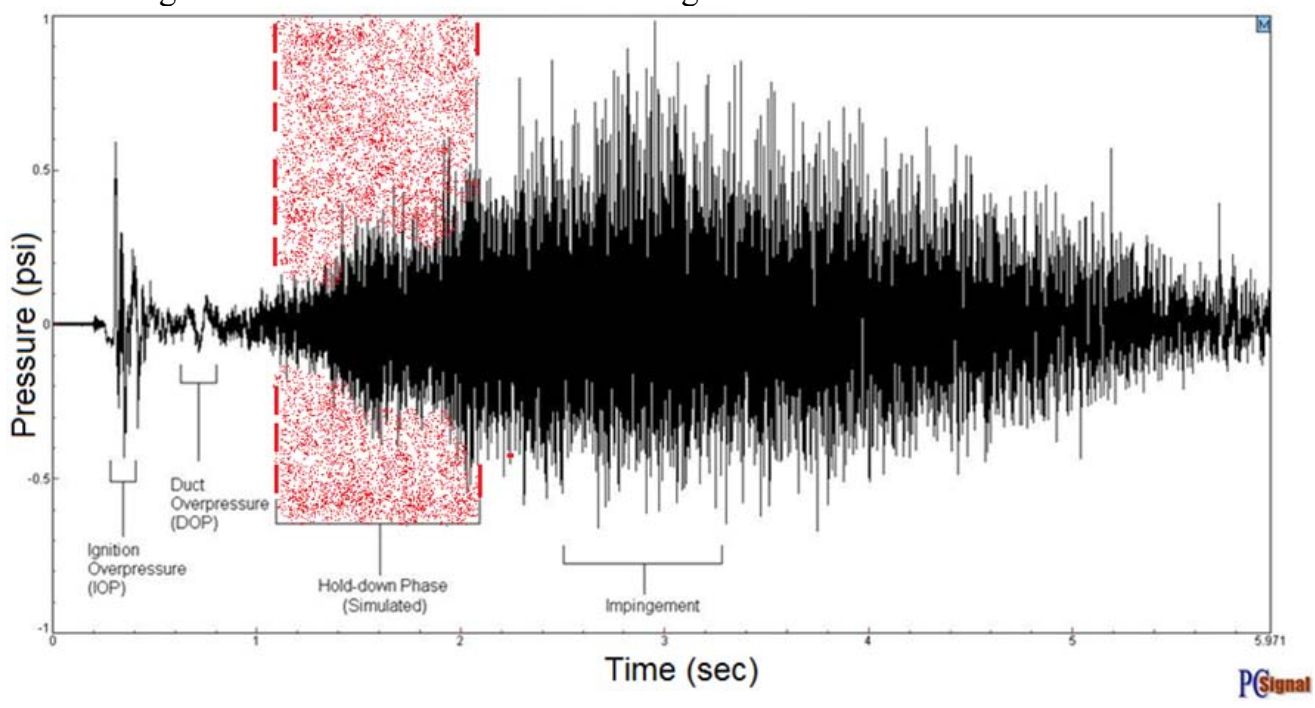

Figure 7. Time History of Acoustics Events at Liftoff (aft skirt) 
The data processing methods introduced for sensor AAD158P at the aft skirt was repeated for each relevant sensor on the Ares I-X. Ultimately, four sensors were selected based on their station numbers (location) and the overall quality of the data. The processed data of the selected sensors and their respective locations on the Ares I-X are shown below in Fig. 8.
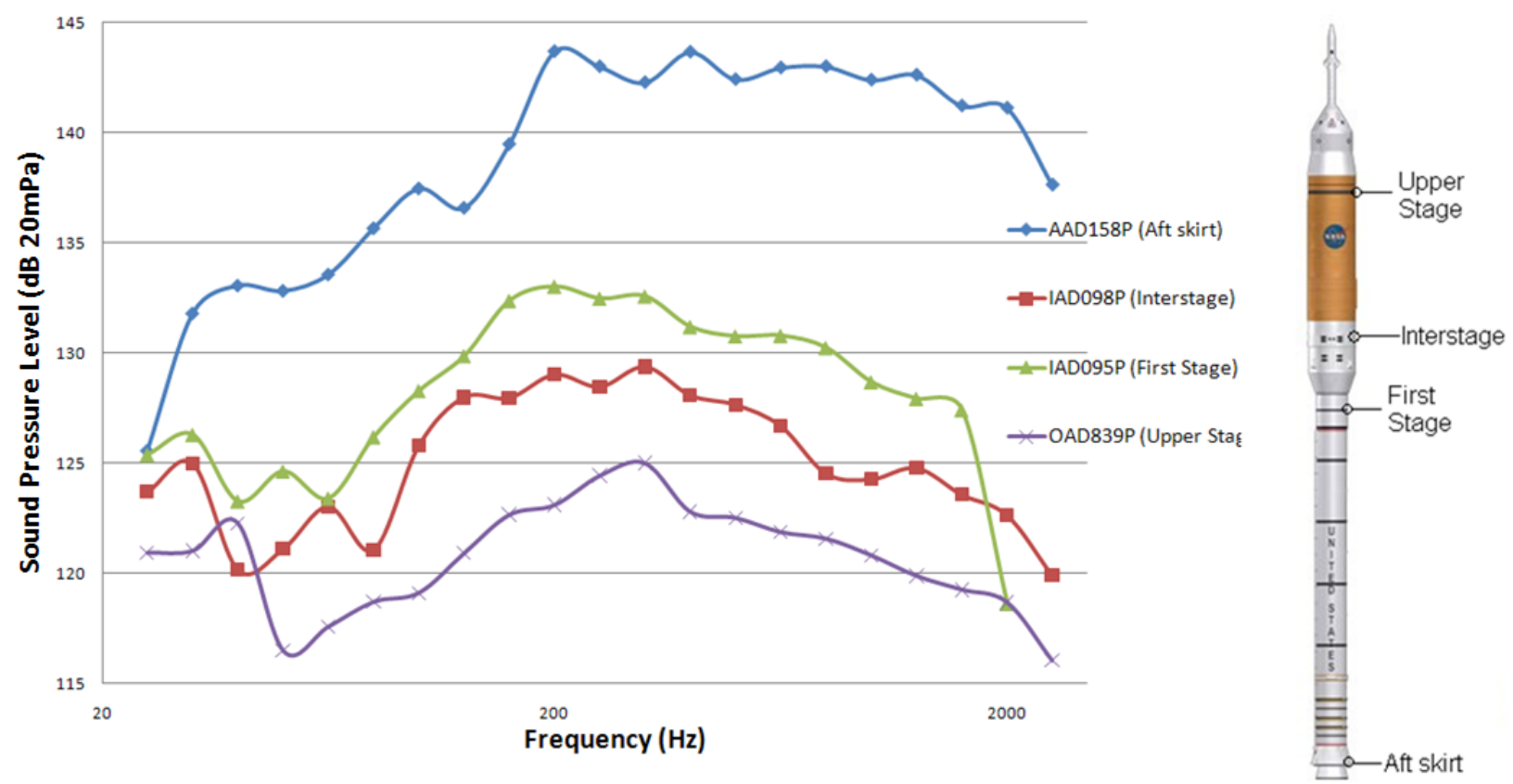

Figure 8. Processed Raw Data of Selected Sensors and their General Locations

As expected, sensor AAD158P displays the highest SPL values due to its location at the aft skirt which represents the nearest distance from the source. It can also be observed that the magnitude of SPL is expected to decrease as the distance from the source increases. One unique phenomenon shown in the raw data presented in Fig. 8 is the sudden increase in SPL at lower frequencies below the $80 \mathrm{~Hz}$ frequency band; a phenomenon present in every sensor except the aft skirt. These low frequency level increases were observed to be residual of duct overpressure (phenomenon displayed in Fig. 7) and factored out of the analysis to avoid significant over prediction due to its nature as an outlier. New shifted polynomial curve fits were then generated using the original SPL values shown in Fig. 8 to allow for the development of smooth DSF and DSL curves for predictions.

\section{Hold-down Phase Dimensionless Source Location (DSL)}

To facilitate a more accurate analysis of the Ares I-X flight data, in addition to improving upon the reliability of data used for future predictions, new dimensionless source locations (DSL) were developed based on the Ares I-X flight data instead of utilizing the same DSL data points related to previous data from static tests such as the J-2, SII, and DYER engines. The DSL can be calculated through a difference between two source locations and their corresponding SPL. As previously discussed, the SPL is proportional to the Dimensionless Source Function (DSF) (which is itself proportional to sound power), the distance the noise source to observation point " $\mathrm{R}$ ", and various scaling parameters such as thrust and effective diameter, as was previously introduced in Eq. 1 where:

$$
R=X+X_{0}
$$

and $\mathrm{X}$ is equivalent to the station number while $X_{0}$ represents the distance from the source to nozzle which itself is equivalent to the DSL divided by the effective diameter.

Based on the fact that the scaling parameters remain constant for the same launch system and the fact that a single overall DSF curve is utilized in the calculation of SPL, a difference between two SPL values will depend solely on the noise source to observation point " $R$ " as shown in Eq. 6 below:

$$
S P L_{1}-S P L_{2}=-20 \log _{10} R_{1}+20 \log _{10} R_{2}
$$


Simplifying the above equation and substituting in Eq. 5 results in an ultimate ratio relation between $\mathrm{R}$ and the change in SPL as shown below:

$$
\frac{X_{2}+X_{0}}{X_{1}+X_{0}}=\sqrt{10^{\frac{\Delta S P L}{10}}}
$$

A DSL generator was written in MATLAB based on Eq. 7 to solve for $X_{0}$ and determine the DSL curves between sensors AAD158P and IAD095P as well as AAD158P and OAD839P. The results were plotted in terms of Strouhal number and displayed in Fig. 9. The DSL curve generated by the AAD158P/IAD095P combination displayed three outlier points, presumably as a result of the polynomial fit taken over the original SPL curve which ignored the three first points of the plots displaying undefined increases in SPL at low frequency. To counter this artificial generation of outliers, the first three points for the AAD158P/IAD095P DSL curve were extrapolated from the fourth coordinate value. Calculating two DSL curves based on separate changes in SPL allowed for a average DSL curve to be generated for use in determining the DSF and ultimately, predictions in SPL. The average DSL curve ranged from a magnitude of 1.4 to 4.4 .

\section{Hold-down Phase Dimensionless Spectrum Function (DSF)}

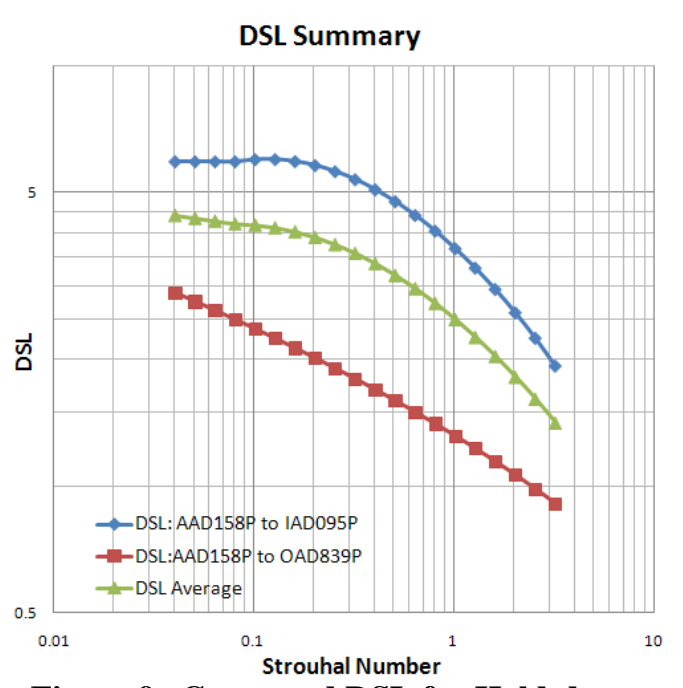

Figure 9. Generated DSL for Hold-down

With the range of DSL curves defined and the data processed in the form of sound pressure levels, DSF curves can be generated for each sensor by rearranging the parameters in Eq.1 as shown:

$$
D S F=\frac{2 * R^{2}\left(10\left(\frac{S P L}{10}-12.75\right)\right)}{\left(2.685 * T T * d_{e}\right)}
$$

The DSF curves for each individual sensor were generated in MATLAB based on Eq. 8 and shown in Fig. 10. To

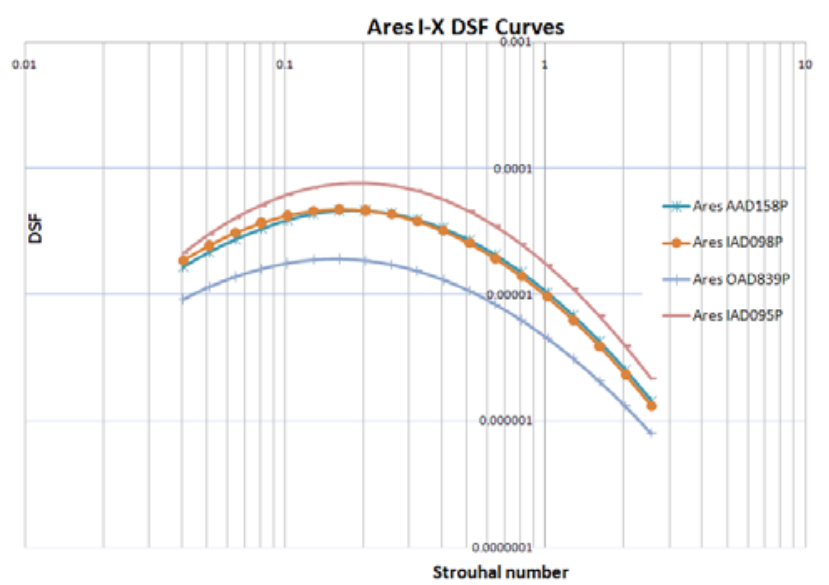

Figure 10. Generated DSF for Hold-down allow for semi-empirical predictions of sound pressure levels, an "overall" DSF curve must be generated from the four individual sensor dependent curves to be referenced by PAVE. To achieve this requirement, multiple generation methods were explored and tested.

\section{Envelope Analysis}

Initially perceived methods for generating an overall DSF assumed that the sensor displaying highest DSF magnitudes will be selected in order to "envelope" all other DSF curves and account for the worst possible scenario. This method was first experimented by using the generated DSF for sensor IAD095P as the base for SPL semi-empirical predictions carried out by the PAVE program. To determine the reliability and accuracy of the DSF envelope method, an prediction analysis was carried out on PAVE where the SPL value for each of the four selected sensors were predicted based on the launch conditions of the Ares I-X and the DSF data of sensor IAD095P. The results were then compared to the original processed SPL data and plotted in Fig. 11.

Observation of Fig. 11 shows that the PAVE program over predicts every sensor except for the base sensor it uses (IAD095P). Over predictions are highest in locations nearest to the source, (in this case, sensor AAD158P at the aft skirt) where the predicted curve is higher by $10 \mathrm{~dB}$. Other comparisons excluding IAD095P were over predicted by about $5 \mathrm{~dB}$. Needless to say, these values are far too high to be considered reliable let alone, realistic, and prove that the envelope method may not be the best choice for generating an overall DSF curve. Furthermore, it 
can be understood that the highest DSF values (as was shown by IAD095P in Fig. 10) do not directly correlate to the highest SPL values as was originally perceived (as shown by IAD095P in Fig. 11).

Averaging Method 1: Averaging Sensors at Highest and Lowest Station Numbers

As it was previously concluded that an enveloping method for overall DSF curve generation is not suitable and severely over predicts, new averaging methods were developed utilizing individual DSF curves. The first method utilized the average between the DSF of sensor AAD158P at the aft skirt (lowest

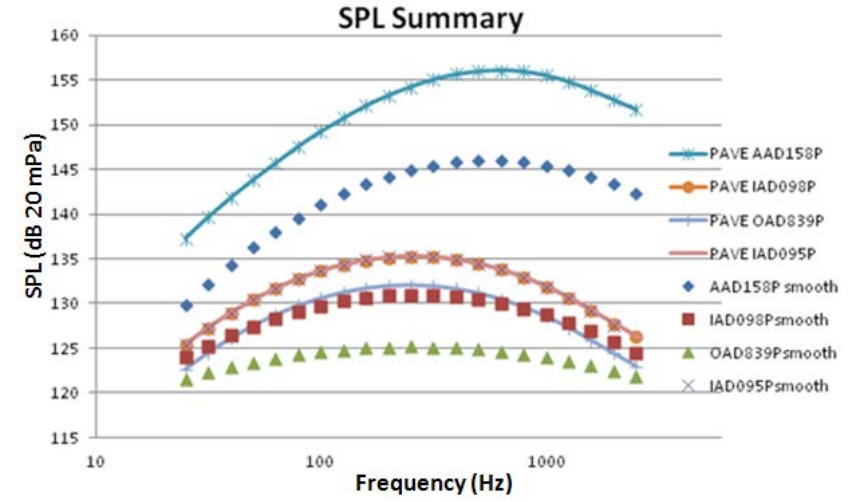

Figure 11. Enveloping DSF Accuracy (Hold-down) station number) and that of OAD829P (highest station near capsule). The resultant DSF curves and calculated average are displayed in Fig. 12. The prediction results are better than the enveloping method used previously, yet because the average was selected between the sensor located at the highest station number (AAD158P) and lowest station number (OAD839P), PAVE tended to under-predict the AAD158P sensor and over-predict OAD839P. This result is undesirable as AAD158P represents the aft skirt region where the highest SPL values exist and where many important instrumentation are installed. An analysis of the IAD sensors show another unique but relatively undesirable situation. The predictions generated by PAVE for IAD095P and IAD098P almost directly overlap each other. This error is a consequence of the PAVE program's 1-D apparent source nature. PAVE generates its

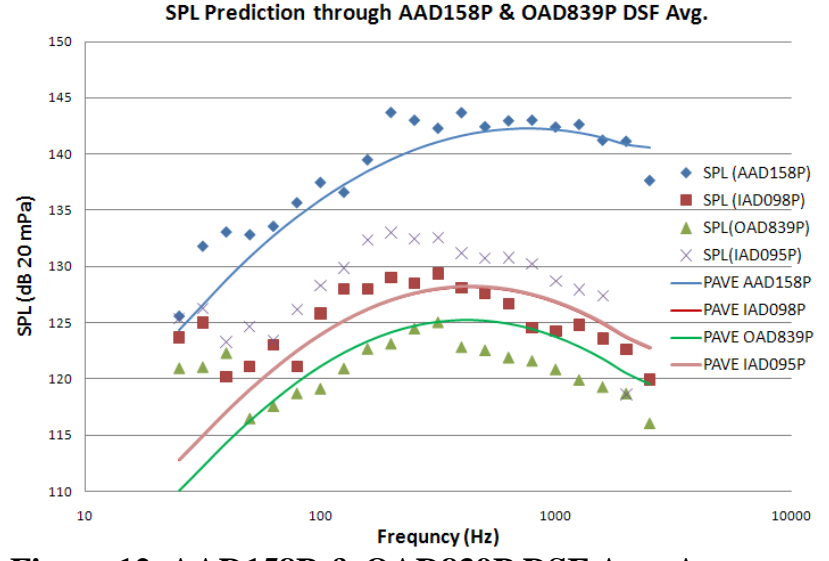

Figure 12. AAD158P \& OAD839P DSF Avg. Accuracy (Hold-down) predictions through the SPL equation (Eq.1) which is primarily dictated by the DSF and DSL which itself is dependent on station number. The two IAD sensors are only $0.6 \mathrm{ft}$. apart from each other in terms of station number. However, the raw data shows that a difference of around 3 to $4 \mathrm{~dB}$ still exists between the IAD sensors. Specifically, while the IAD sensors are positioned at nearly the same station number on the Ares I-X, they are not at the same angle. The IAD095P was positioned at approximately 88 degrees while the IAD098P was positioned at the furthest out location at 0 degrees. However, the PAVE program does not compute angle values in its semi-empirical predictions. It is a 1-D program, and since the station numbers between the two sensors are identical, and all other input values are the same (DSF, thrust, diameter, etc.), the predicted SPL for both sensors will be mathematically identical. Given PAVE's 1-D nature, this issue is bound to be unavoidable for the IAD sensor combination. In order to compensate for these prediction errors, it would be ideal to generate a new averaging method that increases these overall curves. This will not only prevent under-prediction at the aft skirt but will also shift the IAD curves to a more suitable and average location.

\section{Method 2: Averaging All Sensors at Excluding Aft Skirt (AAD185P)}

As the first averaging method tended to under predict several of the sensors and most importantly, the aft skirt sensor, AAD158P, the decision was made to remove AAD158P from the average of the generation of the overall DSF curve primarily due to its significantly higher SPL ranges. The average DSF was taken among both IAD sensors at the first and interstages of the rocket, and the OAD sensor at the upper stage, and tested for predictions accuracy in PAVE. The resulting SPL curves generated by PAVE using the averaged curve are plotted and compared to the raw SPL data in Fig. 13. Results show much more promising and accurate values than the first averaging method. Most importantly, PAVE provided a smooth prediction for sensor AAD158P at the aft skirt where the greatest SPL values are present. As was with the previous methods, the SPL's predicted by PAVE for the IAD sensors are identical and nearly overlap each other. However, the curves trended higher when compared to method 1 and serve more as an averaged SPL curve between the IAD sensors and account for both conditions. The 
generated SPL curve for IAD095P under predicts the SPL within a range of $2-3 \mathrm{~dB}$ which can be considered acceptable while the over prediction range for IAD098P is even lower at 1-2 dB. PAVE over predicts sensor OAD839P (located near the capsule) by an average of $3 \mathrm{~dB}$ which can be considered tolerable as well. Ultimately, Averaging Method 2 shows promising results as a potential overall DSF curve that can be utilized for future acoustic environment predictions for the RSRM configuration.

\section{B. Liftoff Phase}

As currently existing methods for predicting SPL values utilize stationary data from previously conducted hold-down tests, the effects of impingement at liftoff are not accounted for and

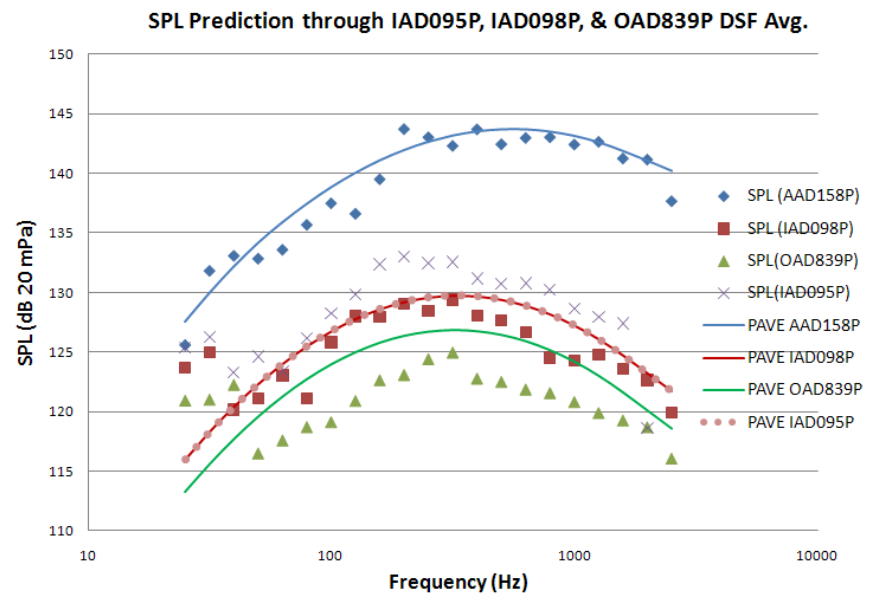

Figure 13. IAD and OAD DSF Avg. Accuracy (Hold-down) consequently result in under predicted conditions that would require the addition of a correction factor. Specifically, impingement occurs moments after ignition when the rocket begins to leave the pad. The plume will no longer be isolated within the trench area beneath the launch pad and will spread across the pad where its energy is radiated back towards the rocket. Such conditions have led to incentives to generate DSF and DSL curves based on Ares I-X flight data covering the entire liftoff phase that would already have the effects of impingement factored into the data as the flight data itself is inherently non-stationary.

Throughout the period of an entire launch, different frequencies peak at different times, thus processing the data in terms of liftoff requires a different approach from the simulated hold-down data where a specific time frame was selected for a specific scenario. In order to define the SPL conditions of the liftoff phase which include the maximum levels caused by impingement, all 1/3 octave bands must be considered as shown in Fig. 14.

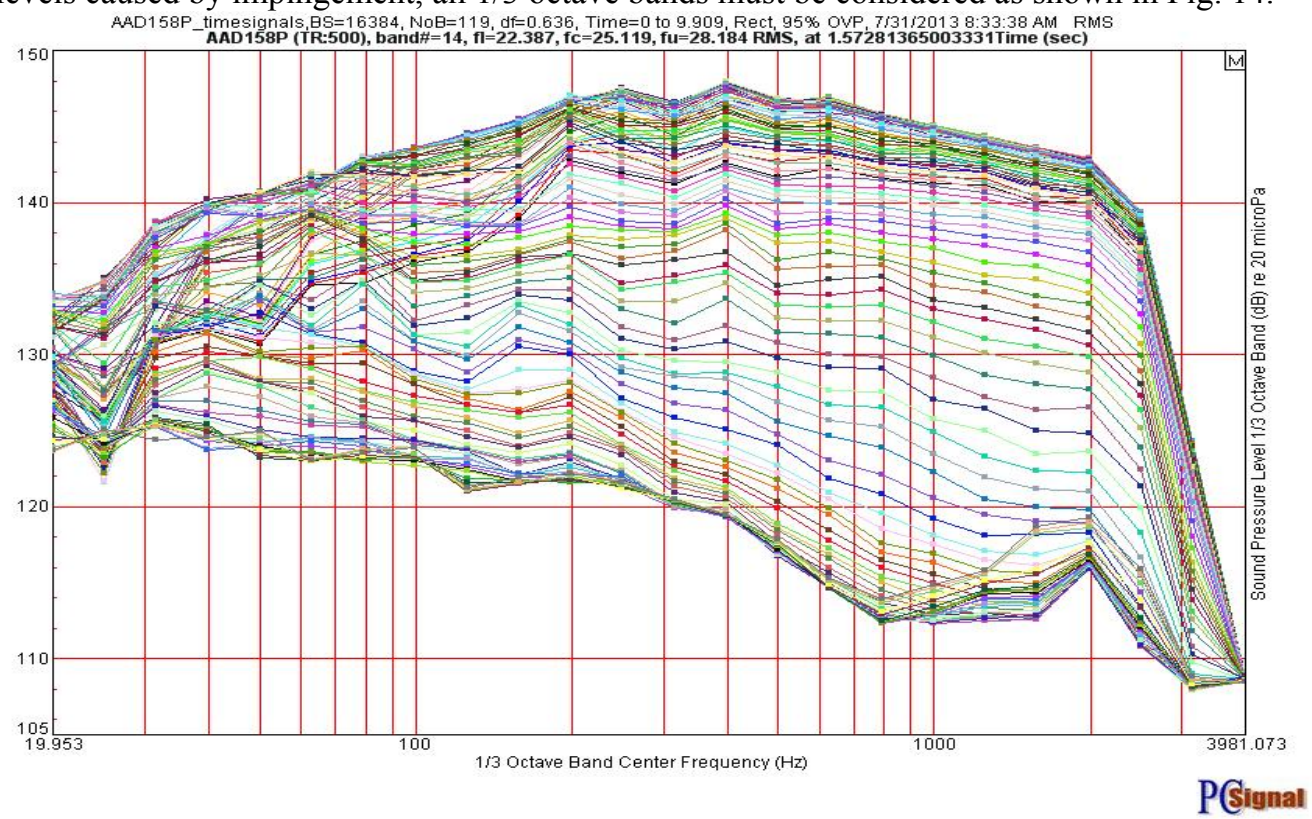

Figure 14. SPL magnitudes with respect to all 1/3 Octave Bands (aft skirt)

Ultimately, the maximum SPL must be extracted from each 1/3 Octave Band, irrespective of time. This "artificial" spectrum is known as a peak hold spectra and is displayed in Fig. 15 below. 

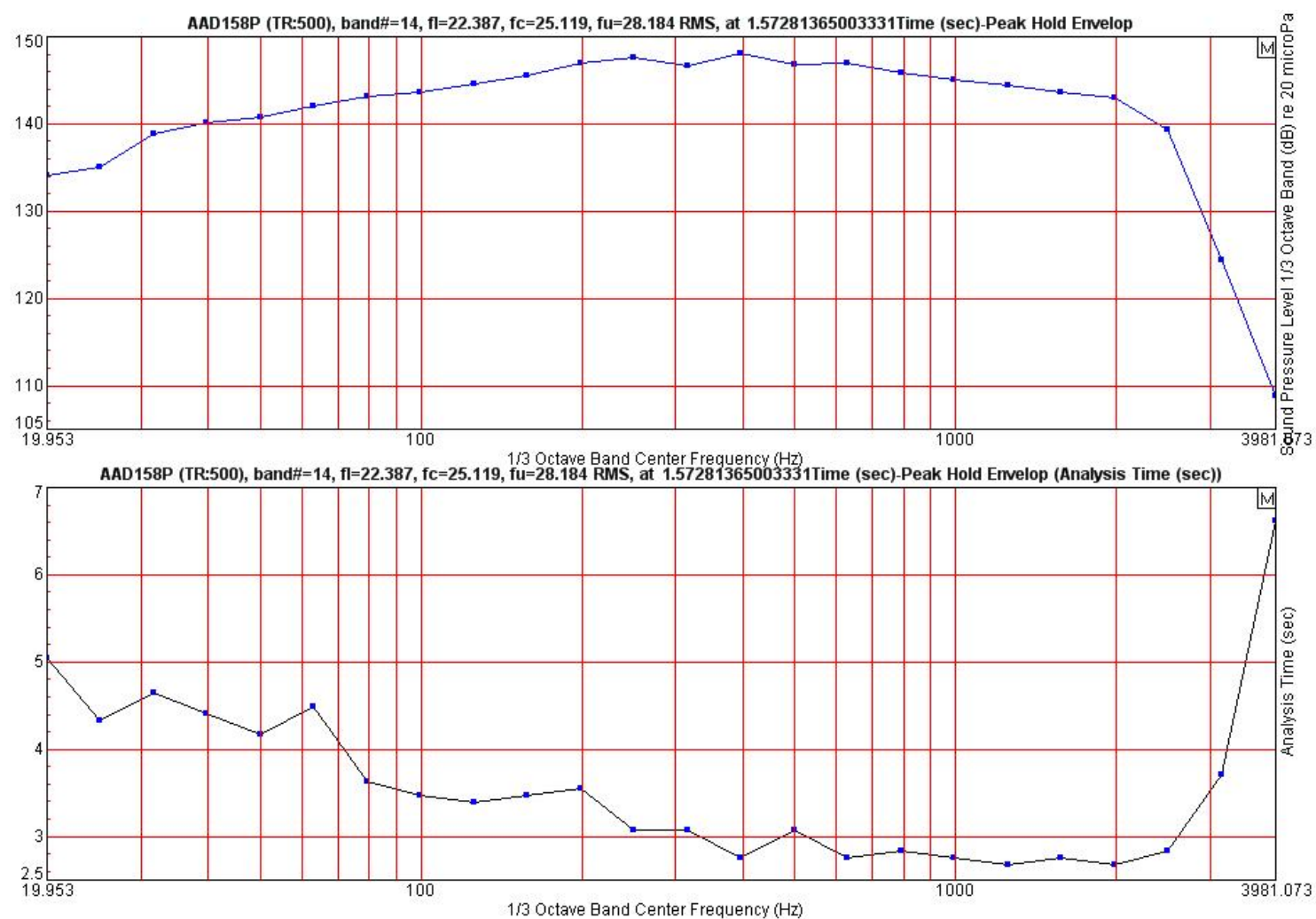

Figure 15. Peak Hold Spectra (Above) and Time of Peak Occurrence (Below) for aft skirt

Fig. 15 shows that higher frequencies tend to peak at an earlier time than lower frequencies which is in agreement with the fact that acoustic events that induce the highest frequencies, such as impingement, occur at an earlier stage of liftoff (a effect that was also previously observed in Fig. 7). Individual peak hold spectras were processed for each of the same four sensors used to develop the hold-down curves and utilized as raw data for the development of DSF and DSL curves that cover the entire liftoff phase of the Ares I-X.

\section{Liftoff Phase Dimensionless Source Location (DSL)}

Similar to the requirements of the hold-down DSF curve generation process, a new set of DSL curves was generated for the liftoff phase. Using the same methods as the hold-down phase, DSL curves were generated between sensors AAD158P and IAD095P as well as AAD158P and OAD839P. As an abnormal increase in magnitude occurs at lower Strouhal numbers for the AAD158P/OAD839P DSL curve, the overall DSL utilizes a fit from the AAD158P/IAD095P curve for the lower half Strouhal numbers before taking an average between both DSL curves. The results were plotted in terms of Strouhal number and displayed in Fig. 16 and compared to the previously generated DSL curve for the simulated hold-down phase. It can clearly be seen that the DSL values generated for the entire launch covered a much higher range than that of the hold-down phase. This is ultimately a consequence of the methods used to obtain the SPL values for the entire launch phase. SPL values were obtained from a

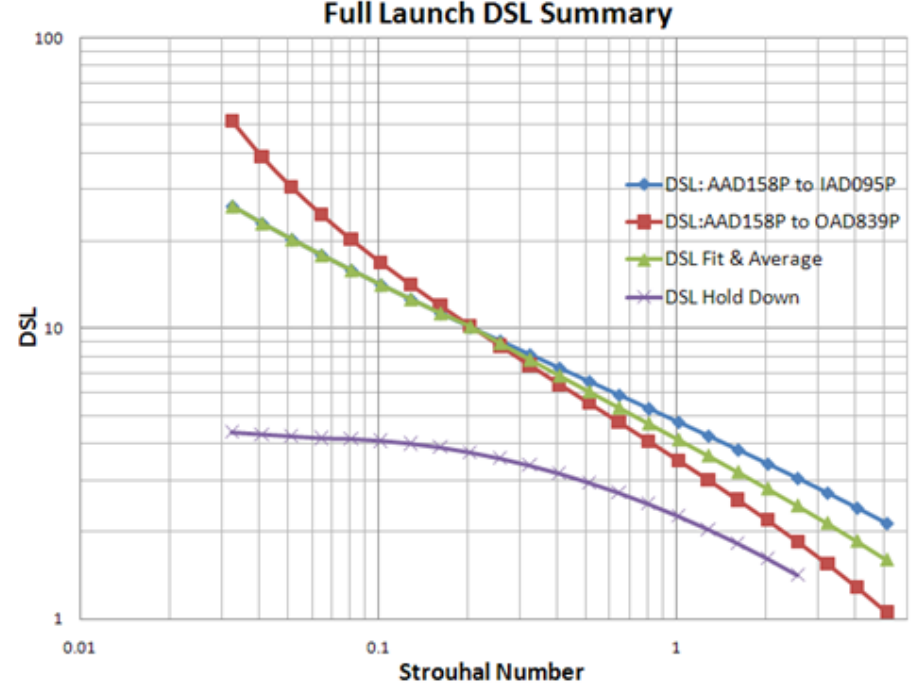

Figure 16. Generated Liftoff DSL Compared to Hold-down 
peak hold spectrum which was described previously as an "artificial" spectrum irrespective of time. Specifically, maximum values were selected from every frequency that exists in a channel, regardless of the time these peaks may have occurred. However, the lift off phase of the Ares I-X was relatively short (within 5 to 6 seconds). Thus a change in time would have had a significant effect on source location. Consequently, the randomness in time ranges when obtaining the peak hold spectra would result in a wide range of source locations as one maximum may have been recorded when its source location was still near the pad while another may have been recorded when the source location has cleared the pad.

\section{Liftoff Phase Dimensionless Spectrum Function (DSF)}

With an overall DSL curve generated for the liftoff phase, individual DSF curves were generated for each sensor using the same methods previously discussed for the hold-down phase. In addition, an overall DSF curve was generated using the same averaging method as the simulated hold-down phase. The individual and overall DSF curves are presented in Fig. 17. Due to the DSF's original peak hold nature where the maximum SPLs were extracted from a wide frequency range, the resultant DSF curves are less distributed when compared to those generated for the simulated hold-down phase. To validate the accuracy of the overall DSF curve, predictions were made in PAVE using the overall DSF curve and compared to the original processed flight data as shown in Fig. 18.

The consistency maintained along each DSF curve allowed for smooth averaging and resulted in predictions that were even more accurate than those obtained from the simulated hold-down phase. As shown in Fig. 18, the generated overall DSF curve predicts a very smooth fit through the AAD and IAD sensors and only slightly over predicts the OAD sensor. The average difference between the Ares I-X data and predicted values was $0.97 \mathrm{~dB}$ for AAD158P (aft skirt), $1.06 \mathrm{~dB}$ for IAD095P (first stage), $1.24 \mathrm{~dB}$ for IAD098P (interstage), and $1.2 \mathrm{~dB}$ for OAD839P (upper stage).

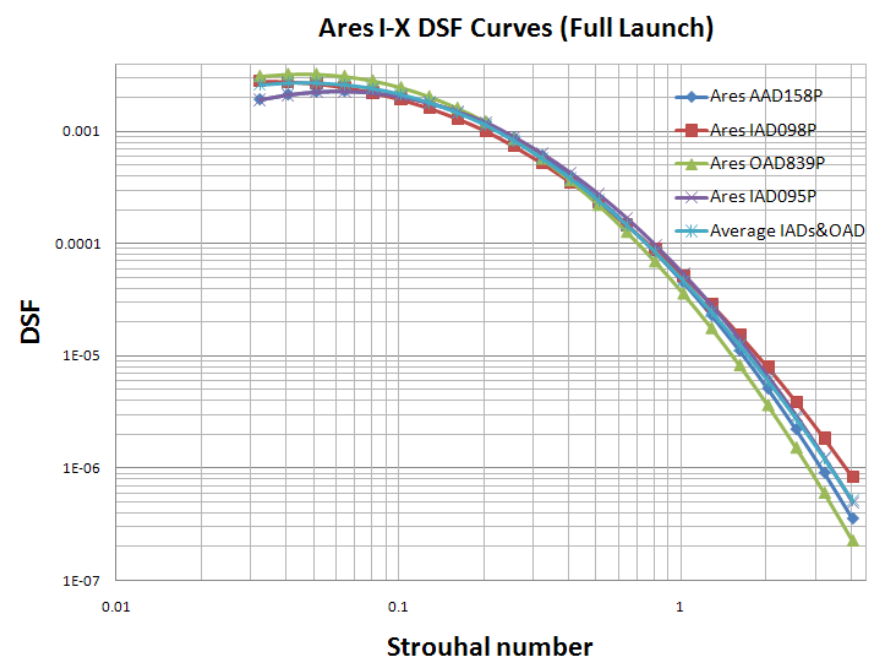

Figure 17. Generated DSF for Liftoff Hold-down SPL Prediction through IAD095P, IAD098P, \& OAD839P DSF Avg.

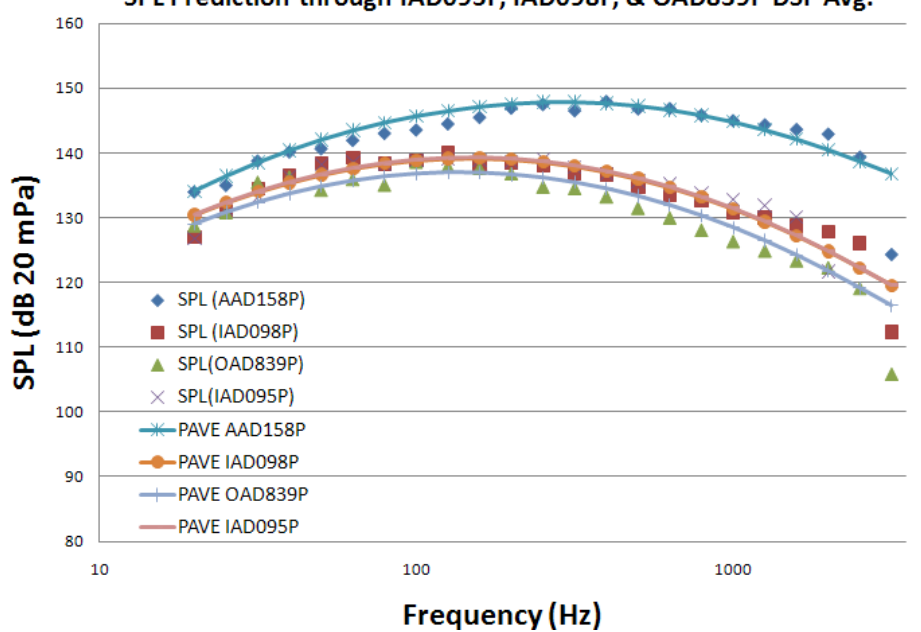

Figure 18. IAD and OAD DSF Avg. Accuracy (Liftoff)

\section{Results and Comparison}

After successfully processing the Ares I-X flight data for both simulated hold-down and liftoff conditions, a series of comparisons and predictions were conducted with multiple static hold-down tests conducted at Marshall Space Flight Center involving both liquid and solid fuel rockets. In addition, the Ares I-X prediction package's capabilities were tested against scaled model test results of the Ares I obtained from the ASMAT program.

\section{A. Dimensionless Spectrum Function Analysis}

To facilitate understanding of the differences between LOA results obtained from non-stationary and stationary data, the Ares I-X hold-down and liftoff DSF curves were plotted together with the DSF curves generated from static tests 
conducted in the 1960's (which were previously used as the primary data sets for LOA loading predictions). The resultant comparison is shown in Fig. 19.

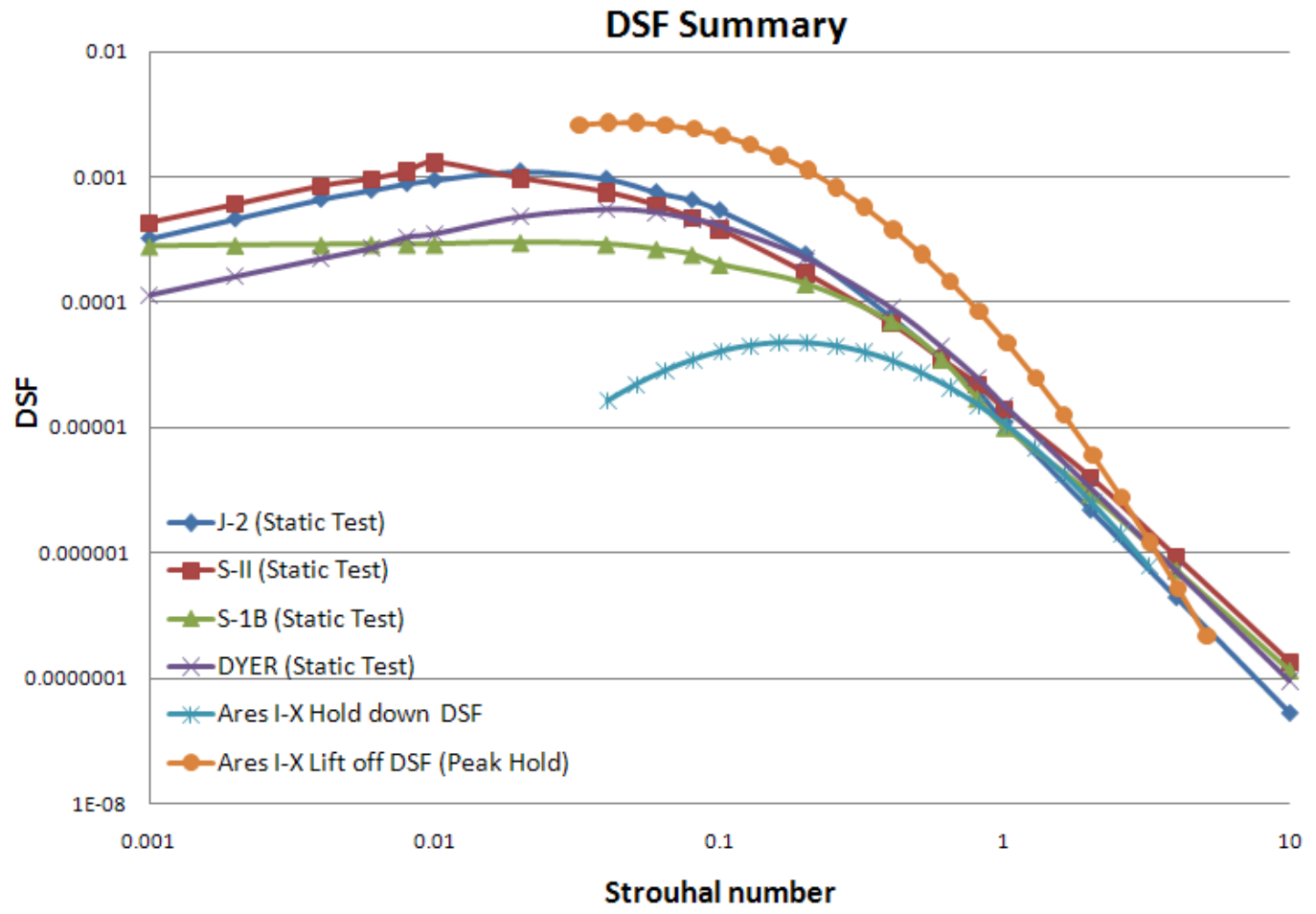

Figure 19. Overall DSF comparison between Ares I-X and previously conducted Static Tests

It can be observed that the simulated hold-down DSF curve for the Ares I-X differs significantly from the static tests, covering a much narrower and lower range in magnitude. This results as a consequence in launch platform configuration and launch conditions. The static hold-down tests were conducted on an open J-deflector system with no side deflector or water cooling system which would have induced higher fluctuations in sound pressure levels.

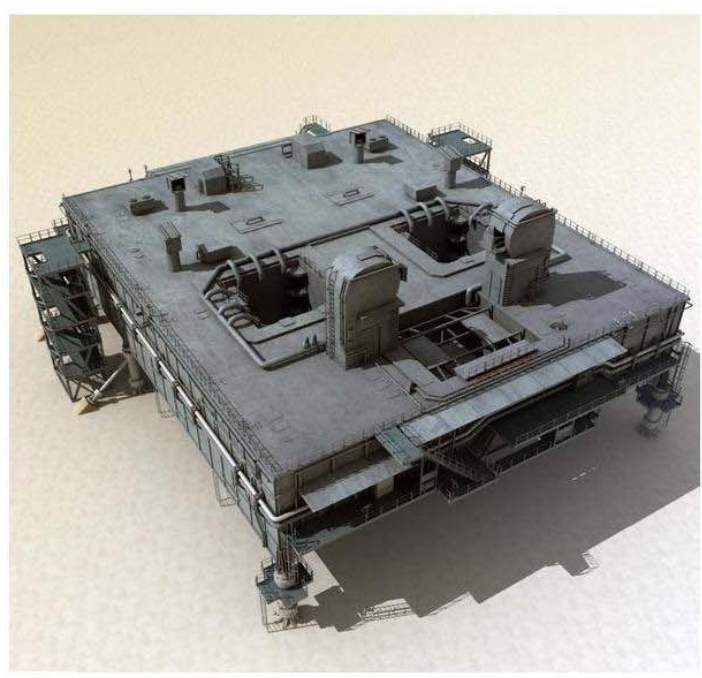

Mobile Launch Platform (MLP) for Ares I-X (Equipped with various damping components including deflectors and Rainbird water system)

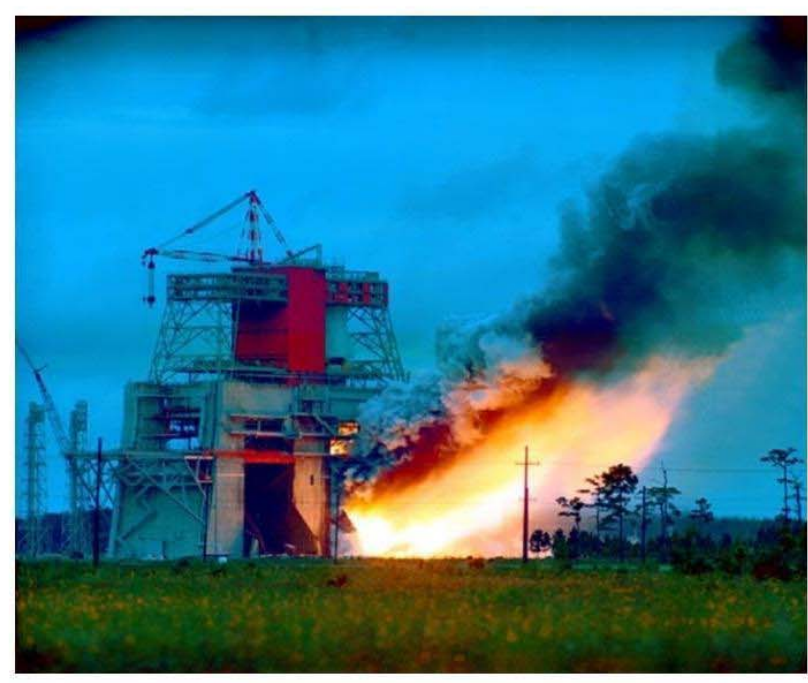

Static Firing Test Stand at MSFC (No Side Deflector or Water System)

Figure 20. The Mobile Launch Platform and Static Firing Test Stands

On the contrary, the Ares I-X was launched from the mobile launch platform which was equipped with side deflectors and water sound suppression systems which would contribute to a decrease in sound pressure and 
reduction of fluctuations. More importantly, the time range selected while processing the simulated hold-down phase was at an earlier stage at lift off where the water suppression systems would have had a more significant effect. Consequently, the resultant simulated hold-down DSF for the Ares I-X can be considered an "isolated" event.

The liftoff curve for the Ares I-X displayed the highest range of DSF values compared to both the simulated hold down and static tests. A number of conditions contributed to the liftoff DSF curve's wide rage. The peak hold curve which was used to develop the liftoff DSF, was generated by selected the maximum from every frequency at various times of launch, which includes the effects of impingement, and inherently covers a wide range in terms of DSF magnitude. In addition, the liftoff curve covers the entire launch time frame. During the liftoff phase, the Ares I-X did not reach the Rainbird water suppression system on the pad and was effectively a "dry launch". Thus the only water suppression systems that had an effect were those installed within the mobile launch platform and trench area beneath the rocket. As the liftoff time of Ares I-X was short, the majority of the liftoff curve will cover distances where the rocket has pulled further away from the water suppression systems and consequently, result in higher values in SPL and DSF.

\section{B. Ares I-X Flight Data and DYER Static Test Data Comparison}

To demonstrate the capabilities of non-stationary data prediction in comparison to stationary data prediction of inherently non-stationary flight conditions, two predictions were carried out in PAVE of the entire Ares I-X liftoff phase with parameters relating to the rocket using stationary data from the DYER hold down tests and Ares I-X liftoff data. The station locations used were selected from the aft skirt, first stage, and upper stage sensors. The results were then plotted and compared against the raw Ares I-X peak hold data in Fig. 21.

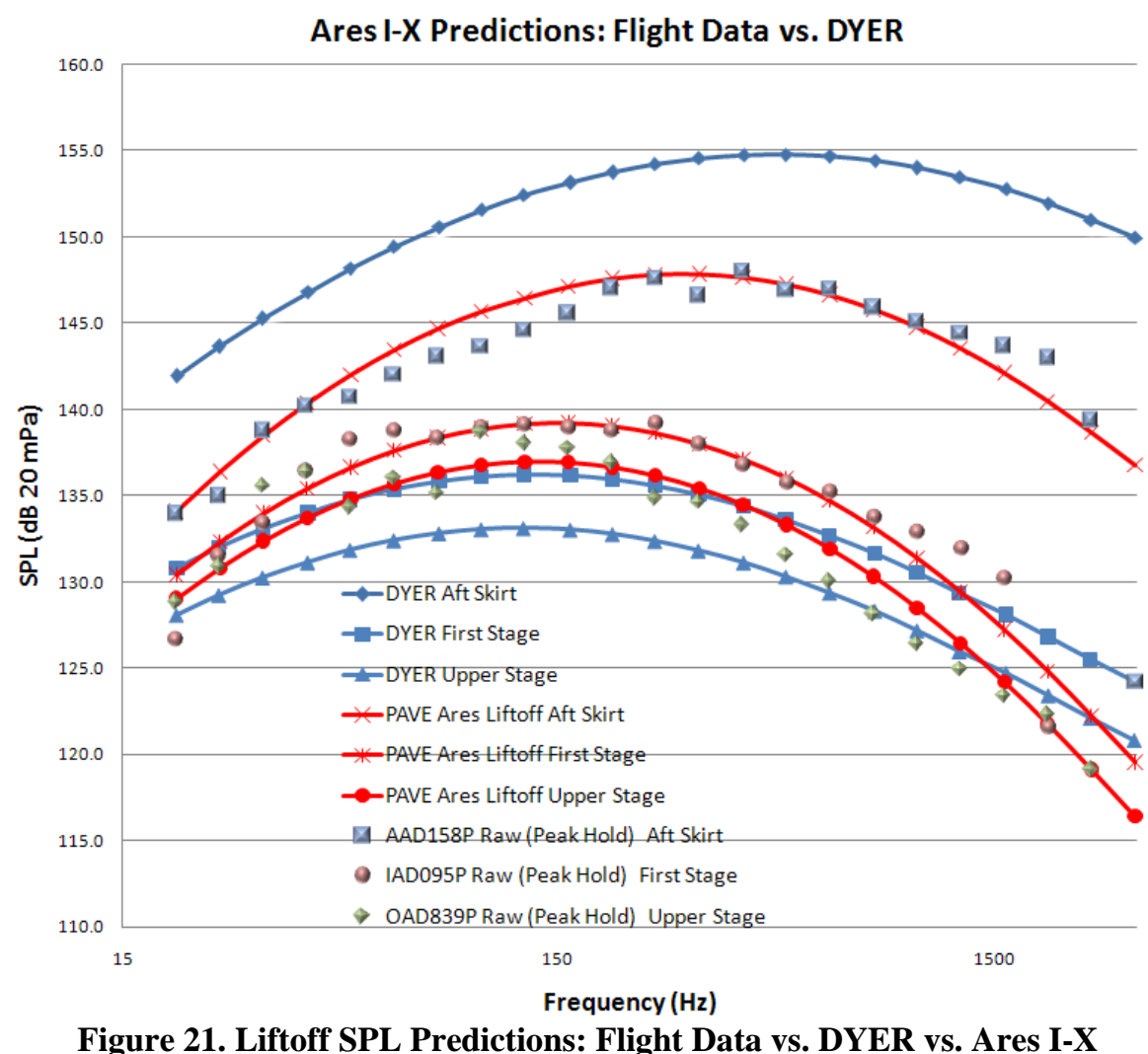

As expected, the stationary data from the DYER tests under predict for a majority of the station locations. DYER tended to under predict the first stage and upper stage regions by an average difference of $3 \mathrm{~dB}$ while over predicting the aft skirt region by $8.8 \mathrm{~dB}$. This correlates to the use of correction factors in past predictions using stationary data to account for the effects of impingement. Predictions using non-stationary Ares I-X liftoff data, as shown in Fig. 21 , fared better and tended to smoothly fit over the raw flight data. The average differences calculated for the aft skirt, first stage, and upper stage regions, were $0.97,1.1$, and $1.2 \mathrm{~dB}$ respectively. As discussed before, the liftoff prediction package developed from the Ares I-X flight data is inherently non-stationary and factors in major 
increases in SPL such as the effects of impingement. Ultimately, the analysis confirms that it is more suitable to use non-stationary data over stationary data in the prediction of non-stationary conditions.

\section{Ares I-X Flight Data and ASMAT Comparison}

The Ares I Scale Model Acoustic Test (ASMAT) program was implemented and executed at Marshal Space Flight Center to verify predicted LOA environments as well as the effectiveness of water suppression systems. The test was conducted utilizing a 5\% model of the Ares I launch vehicle as well as a mobile launch platform equipped with a tower. Multiple hold-down tests were conducted at various elevations to simulate the entire launch sequence of the rocket. As a similar Scale Model Acoustic Test (SMAT) will be conducted for the Space Launch System (SLS) program, it is of interest to test the capabilities of the newly processed Ares I-X prediction package in relation to past data from ASMAT.

Three station numbers were selected at model scale that represented the positions of the original sensors processed from the Ares I-X flight. These locations were measured from the nozzle of the model at $5.06 \mathrm{in}$. (aft skirt), $91.63 \mathrm{in}$. (first stage), and 164.5 in. (upper stage). In addition, two separate tests with similar conditions to the Ares I-X launch were selected to be compared with PAVE predictions. An on-pad ASMAT test with active water suppression was compared to PAVE predictions utilizing the simulated hold-down Ares I-X data while an ASMAT test conducted at $5 \mathrm{ft}$ elevation from the pad without Rainbirds activated was compared to PAVE predictions utilizing the liftoff data. The results for the first stage are displayed in Fig. 22 below.

\section{ASMAT First Stage (91.63 in.)}

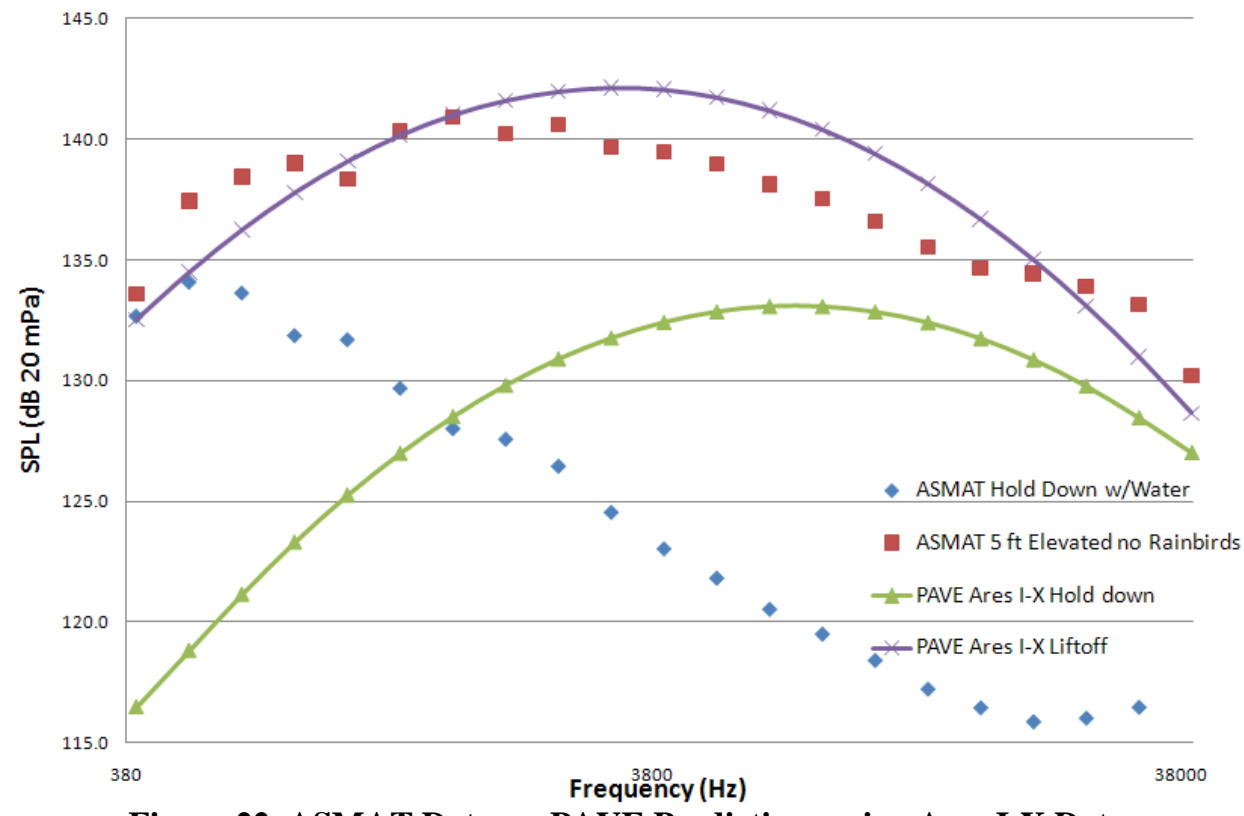

Figure 22. ASMAT Data vs. PAVE Predictions using Ares I-X Data

The average difference calculated between the ASMAT on-pad hold-down test and PAVE predictions using the Ares I-X simulated hold-down condition was $10.5 \mathrm{~dB}$. It is clearly observed in Fig. 22 that PAVE over predicts conditions for ASMAT in the majority of frequencies. The major difference between predicted and measured SPL is possibly a resultant consequence of the launch conditions specific to the Ares I-X rocket. Two forms of water sound suppression systems were applicable at the early stages of the Ares I-X launch. These included the IOP water sprayed from the Mobile Launch Platform (MLP) and deflector water which was sprayed within the trench area (separate from the MLP). As was previously discussed, the Ares I-X cleared the launch complex in a very short amount of time (on the order of 5-6 seconds). The distance between the rocket and the launch platform would increase significantly as time increased which would also increase distance between the nozzle and both the IOP and deflector water suppression systems resulting in a continuous increase in pressure levels; an effect that was clearly visible in the time history of the simulated hold-down phase previously introduced in Fig. 7. As previously discussed, Ares I-X was considered a "dry launch" as the Rainbird water suppression system had no significant effect as the rocket lifted from the launch complex and pulled away from the water suppression systems. The time range selected for the Ares I-X simulated hold-down phase was at 1.5 to 2.5 seconds, where the rocket was 
effectively about 20 feet above the pad. The ASMAT model however was held in a stationary position during testing allowing both the IOP and deflector water systems to significantly suppress the sound pressure levels over time.

PAVE predictions based on the entire liftoff phase showed more promising results. The predicted values were compared to a similar ASMAT test simulating a dry launch scenario at a $5 \mathrm{ft}$. elevation as shown in Fig. 23. The average difference calculated between the ASMAT elevated dry launch test and liftoff predictions by PAVE using Ares I-X flight data was $1.8 \mathrm{~dB}$ at the comparable first stage location. The scenario experienced by the ASMAT scaled model is a better comparison to the real Ares I-X launch as the model is much further away from the IOP and deflector water suppression systems while impingement conditions start taking effect without suppression from Rainbirds. The results from other station numbers at both the aft skirt and upper stage tended to mimic the pattern of Fig. 22. Specifically, the hold-down average difference was calculated to be $11.7 \mathrm{db}$ for the aft skirt and $9.1 \mathrm{~dB}$ for the upper stage, while the liftoff average difference was $3.4 \mathrm{~dB}$ for the aft skirt and $2 \mathrm{~dB}$ for the upper stage. Ultimately, it can be concluded that launch conditions in terms of the platform and rocket severely impact LOA predictions. Thus the comparison with ASMAT data confirms that for future scaled model tests, the Ares I-X prediction package is more suitable for elevated hold-down conditions than on-pad conditions.

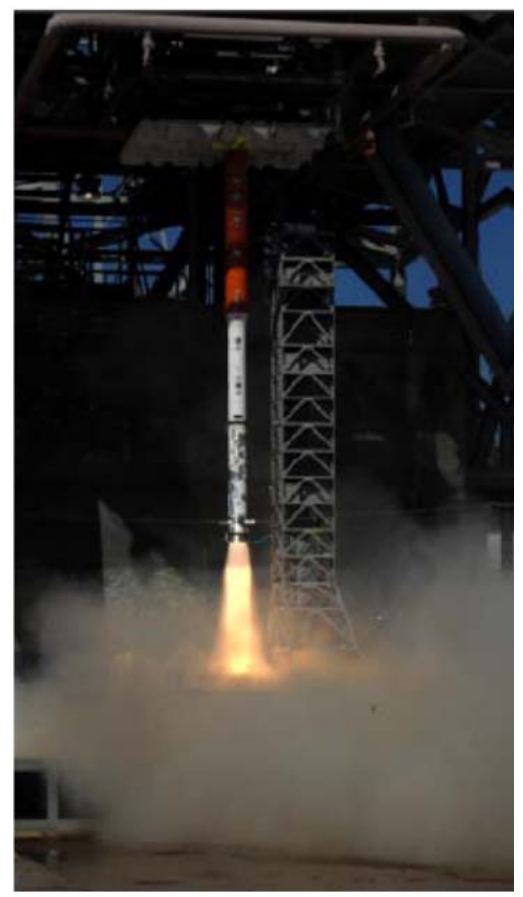

Figure 23. ASMAT Model elevated to $5 \mathrm{ft}$.

\section{Conclusion}

The development of the PAVE program and the Ares I-X prediction package provides a new set of predictive capabilities for determining the liftoff acoustic loadings of current and future launch vehicle programs. The results from the Ares I-X flight prediction package conclusively show that flight data can be accurately reproduced and scaled. Thus the use of flight data can lead to more accurate predictions in acoustic loads, specifically for nonstationary conditions. A comparison of DSF ranges for static tests and the Ares I-X launch shows significant differences possibly due to the differences in launch pad configurations. Additionally, prediction comparisons between stationary and non-stationary based predictions of the Ares I-X launch display more accurate results from the Ares I-X prediction package than those by DYER static tests which tend to under predict. Comparisons with ASMAT data however, show that the Ares I-X prediction package is not suitable for on-pad hold-down conditions as the effects of a constant distance between the nozzle and water suppression system induces a bias in the data that otherwise, would not occur under real launch conditions. Comparisons with ASMAT conditions do show that nonstationary flight data is suitable for elevated hold-down predictions where the nozzle is at a further distance from the pad. Conclusively, analysis shows that non-stationary and stationary predictions should be kept separate as the launch conditions for the origins of both forms of data are significantly different.

\section{Acknowledgments}

Janice D. Houston- An excellent mentor whom not only strived to improve the intern's technical ability, but also prioritized interpersonal skills. Created numerous opportunities for networking and exposure to a diverse set of fields including data acquisition and signals/processing.

Jared Haynes- Major reference and source for the theoretical concepts of acoustic loading and introduced concepts of SPL and DSF generation.

Jess Jones-Introduced PC-Signal software through multiple tutorial sessions and aided significantly in the processing of Ares I-X flight data.

Doug Counter- Created the Vehicle Acoustic Environment Prediction Program (VAEPP); PAVE's predecessor. Explained the basics of VAEPP's semi-emperical methods which were adopted by PAVE and provided data for processing of Ares I-X flight data. 
Clothilde Giacomini- Introduced basic GUI generation in MATLAB and aided in multiple stages of PAVE's development

Donald Nance- Provided resources and techniques for overall DSF curve generation and analysis of flight data

Matthew J. Casiano- Aided in several programming methods in MATLAB

Magda B. Vargas- Aided in the generation of some basic 1/3 octave band SPL spectra

\section{References}

${ }^{1}$ Bendat, J. S., and Piersol, A. G., Random Data: Analysis and Measurement Procedures, $2^{\text {nd }}$ ed., John Wiley \& Sons, New York, 1986

${ }^{2}$ Eldred, K.M., “Acoustic Loads Generated by the Propulsion System,” NASA SP-8072, 1971.

${ }^{3}$ Wilhold, G.A., Guest, S.H., and Jones, J.H., "A Technique for Predicting Far-Field Acoustic Environments due to a Moving Rocket Sound Source,” NASA, 1963.

${ }^{4}$ Douglas, D. and Houston, J., "Verification of Ares I Liftoff Acoustic Environments via the Ares I Scale Model Acoustic Test," Aerospace Testing Seminar, 1993.

${ }^{5}$ Haynes, J. and Kenny J.R., "Modifications to the NASA SP-8072 Distributed Source Method II for Ares I Lift-off Environment Predictions," AIAA Aeroacoustics Conference, Vol. 1, AIAA, Miami, Florida, 2009, pp. 1-12. 